\title{
Detection and Characterization of Weissellicin 110, a Bacteriocin Produced by Weissella cibaria
}

\author{
Hui-chung Wu ${ }^{1}$, Sirinat Srionnual ${ }^{2}$, Fujitoshi Yanagida ${ }^{3}$, Yi-sheng Chen ${ }^{1, *}$ \\ ${ }^{1}$ Department of Biotechnology, Ming Chuan University, No. 5, De-Ming Rd., Gui-Shan, Taoyuan 333, Taiwan \\ ${ }^{2}$ Department of Biotechnology, Faculty of Agro-Industry, Rajamangala University of Technology Srivijaya, Nakhon Si \\ Thammarat Campus, Thung Yai 80240, Thailand \\ ${ }^{3}$ The Institute of Enology and Viticulture, University of Yamanashi, 1-13-1 Kitashin, Kofu, Yamanashi 400-0005, Japan \\ ${ }^{*}$ Corresponding author: Yi-sheng Chen, Department of Biotechnology, Ming Chuan University, No. 5, De-Ming Rd., Gui-Shan, Taoyuan 333, Taiwan. \\ Tel: +886-33507001, Fax: +886-33593878, E-mail: yisheng@mail.mcu.edu.tw
}

Received: February 10, 2015; Revised: July 25, 2015; Accepted: August 25, 2015

Background: Weissellicin 110 is the only bacteriocin reported in Weissella cibaria up to now. This bacteriocin represents several unique features. This is the first report on the gene sequence that encodes for the bacteriocin.

Objectives: Providing a rapid detection method to isolate the weissellicin 110 encoding gene and determination of the bacteriocin distribution were the objectives.

Materials and Methods: Bacteriocin from $W$. cibaria 860106 was purified and analyzed using mass spectrometry for proteins sequencing. The draft genome sequence of $W$. cibaria 860106 was generated using next generation sequencing. PCR was applied to detect the weissellicin 110 encoding gene.

Results: The molecular weight and partial protein sequence were obtained for the bacteriocin from W. cibaria 860106. An open reading frame (ORF) was identified as weissellicin 110 from the draft genome sequence. PCR primers were designed to amplify the weissellicin 110 encoding gene and these primers detected sequences from other 27 BLIS-producing $W$. cibaria strains previously isolated from either various Taiwanese fermented foods or the respective raw materials.

Conclusions: The genetic information of weissellicin 110 was obtained, enabling rapid detection of the weissellicin 110 encoding gene. Results suggest that weissellicin 110 producing $W$. cibaria strains are widely distributed inTaiwanese fermented foods.

Keywords: Bacteriocin; Lactic acid bacteria; Weissella cibaria

\section{Background}

Bacteriocins are natural peptides produced by bacteria that display antimicrobial activities. Much recent research has focused on the bacteriocins produced by lactic acid bacteria (LAB). These peptides have potential use as food preservatives (1-3). The classification of LAB bacteriocins has been addressed in many schemes (4-5). Generally, lantibiotics (containing post-translationally modified amino acids such as lanthionine and $\beta$-methyllanthionine) are assigned to Class I (5). ClassII bacteriocins are defined as non-lanthionine-containing bacteriocins and are further subdivided into 4 groups (classes IIa, IIb, IIc, and IId) (5). Many bacteriocins are synthesized as precursors containing leader peptides. Michiels et al. (6) demonstrated the secretion of some bacteriocins requires the activity of a dedicated ATP-binding cassette (ABC) transporter. The activity of this type of transporter typ- ically includes cleavage of the pre-bacteriocin to release a leader peptide (the double-glycine leader sequence) and subsequent translocation of the mature bacteriocin across the cytoplasmic membrane.

Bacteriocins have been frequently reported from Lactobacillus, Enterococcus, Lactococcus, and Leuconostoc $(3,7)$. However, relatively little information is available about bacteriocins from Weissella. A limited number of bacteriocins, such as weissellicin 110, weissellin A, weissellicin Y, weissellicin M, and weissellicin $\mathrm{L}$, have been reported previously from Weissella spp. (8-12). The lengths of these bacteriocins range from 42 to 52 amino acid residues and these sequences are highly diverse, thus it is impracticable to design degenerate primers to amplify the bacteriocins of Weissella spp. Among these, weissellicin 110 is the only bacteriocin reported in Weissella cibaria. This bacteriocin represents several unique features; it is sta- 
ble after high-temperature treatment and has a arrow spectrum of inhibition of other LAB. Additionally, unlike most class II bacteriocins produced by LAB, weissellicin 110 had no activity against Listeria monocytogenes. However, the genetic information was not available in the previous study (8). W. cibaria 860106 , isolated from the yan-dong-gua (fermented wax gourd), is able to produce a bacteriocin but not fully characterized (13). In this study, we identified the $W$. cibaria 860106 bacteriocin as weissellicin 110 .

\section{Materials and Methods}

\subsection{Bacterial Strains and Culture Conditions}

Weissella cibaria strains 860106 and 110, and the main indicator strain Weissella paramesenteroides BCRC $14006^{\mathrm{T}}$, were grown in de Man Rogosa and Sharpe (MRS) medium (BD, Franklin Lakes, NJ, USA) at $30^{\circ} \mathrm{Cs}$ under aerobic conditions without shaking.Inhibitory activity was determined using agar-well diffusion assay described by Srionnual et al. (8). The same culture conditions were used for the propagation of another $19 \mathrm{~W}$. cibaria strains isolated from fermented ginger (2), 5 isolated from fermented cummingcordia or its cummingcordia (14), and 3 isolated from pickled peaches (15).

\subsection{Bacteriocin Purification}

The supernatant containing the bacteriocin was initially harvested using the method described by Yang et al. (16). The harvested cell-free supernatant was desalted and purified using a $\mathrm{C}_{18}$ cartridge (Sep-Pak $\mathrm{C}_{18}$; Waters, Milford, MA, USA) following Chen et al. (17). Antimicrobial activity of the peptide fractions was tested using agar-spot diffusion assay (18). The bacteriocin activity of each fraction was determined against $W$. paramesenteroides BCRC $14006^{\mathrm{T}}$. The active eluted fractions were freeze-dried and stored at $-20^{\circ} \mathrm{C}$.

\subsection{Mass Spectrometry}

The molecular mass of the purified bacteriocin was determined by matrix-assisted laser desorption/ ionization time-of-flight (MALDI-TOF) mass spectrometry (MS) using a SCIEX QSTAR Elite system (Applied Biosystems, Foster City, CA, USA) with CHCA ( $\alpha$-cyano-4-hydroxycinnamic acid) as the matrix.

\subsection{N-terminal Amino Acid Sequence Analyses}

The partial $N$-terminal amino acid sequence of the purified bacteriocin was determined using an Applied Biosystems protein sequencer (model 494, Applied Biosystems). The amino acid sequence obtained was compared with previously reported sequences in the NCBI database (http://blast.ncbi.nlm.nih.gov/).

\subsection{Genetic Analysis}

The draft genome sequence of W. cibaria 860106 was generated using next generation sequencing (data not shown). All contigs (159 contigs) were submitted to the Rapid Annotation using Subsystem Technology (RAST) Prokaryotic Genome Annotation Server (http://rast.nmpdr.org/) and 2395 protein-encoding genes were predicted. The partial $N$-terminal amino acid sequence, SDKNNVFFQIG was applied to search against the predicted protein-encoding genes and located a gene encoding a peptide containing SDKNNVFFQIG. This result identified a contig sequence of 809258 base pairs containing the complete open reading frame (ORF) and the flanking regions of the putative structural gene of weissellicin 110. Homology search was performed using NCBI BLAST (http://blast.ncbi.nlm.nih.gov/) and showed no detectable sequence homology with the ORF identified here.

\subsection{Weissellicin 110 Gene-Specific PCR}

Since the nucleotide sequences of weissellicin 110 and the flanking regions were obtained from the genetic analysis of $W$. cibaria 860106 as discribed in 2.5. Weissellicin 110-specific oligonucleotide primers were designed from $5^{\prime}$ and $3^{\prime}$ flanking sequences of the weissellicin 110 structural gene sequence using NCBI/ Primer-BLAST tool (www.ncbi.nlm.nih.gov/tools/ primer-blast/). The primer sequences were WC_Bac_F $\left(5^{\prime}\right.$-CTGGGAACATGGACGCAGAA- $\left.3^{\prime}\right)$ and $\mathrm{WC}_{-}$Bac_ R (5'-GGACAAGATCGTATGCCGGT-3'). The oligonucleotides were synthesized by Genomics BioSci and Tech Ltd. (New Taipei City, Taiwan). Amplification was started with an initial denaturation for $3 \mathrm{~min}$ at $95^{\circ} \mathrm{C}$ that followed with 30 cycles of $30 \mathrm{sec}$ at $95^{\circ} \mathrm{C}, 30$ sec at $61^{\circ} \mathrm{C}$, and $90 \mathrm{sec}$ at $72^{\circ} \mathrm{C}$; and a final extension for $10 \mathrm{~min}$ at $72^{\circ} \mathrm{C}$. The PCR products were visualized on a $2 \%$ agarose gel in $1 \times$ TAE. A 100 bp DNA ladder marker (Genomics BioSci and Tech Ltd., New Taipei City, Taiwan) was used as the size standard. Besides $W$. cibaira 860106, strain W. cibaria 110 (8) was used as the positive control. Strains $W$. hellenica 4-7 (weissellicin L producer) (11) and W. cibaira I042209 and I042210 (no bacteriocin-producing ability) (19) were used as the negative controls. 


\section{Results}

An isolated peak with bacteriocin activity was observed in the second purification step of reversephase chromatography (Figure 1A). Analysis by MALDI-TOF-MS revealed a clear main peak at 3492.21 Da (Figure 1B). To determine the sequence of this peptide, $\mathrm{N}$-terminal amino acid analysis of the purified bacteriocin was performed, yielding the following partial sequence: $\mathrm{NH}_{2}$-SDKNNVFFQIG.

The $N$-terminal sequence was employed to search against a translation of the draft genome of $W$. cibaria 860106 (unpublished). The nucleotide sequences encoding the putative structural gene for this bacteriocin and its flanking region were identified as described in Materials and Methods 2.5 and 2.6 (Figure 2A). The deduced bacteriocin comprised 52 amino acid residues in the full-length precursor pep- tide and 31 residues in the mature peptide. The translated full amino acid sequence of the mature peptide was as follows: $\mathrm{NH}_{2}$-SDKNNVFFQIGKRYVAPVLYWFGKGAEGIKG. The mature 31-amino-acid peptide was predicted to have a molecular weight of 3490.02 $\mathrm{Da}$ using the Compute $\mathrm{pl} / \mathrm{Mw}$ tool on the ExPASy Proteomics Server (http://web.expasy.org/compute pi/), and was consistent with the result obtained by MALDI-TOF-MS.

Comparing W. cibaria 860106 peptide with other Weissella bacteriocins, both the $N$-terminal protein sequence and the molecular weight match weissellicin 110 (8). However, the nucleotide sequence of weissellicin 110 was not reported and no sequence was observed via BLAST. Thus, the sequence determined in this study was defined as weissellicin 110 and deposited in the DDBJ (accession number LC010242; (Figure 2A).
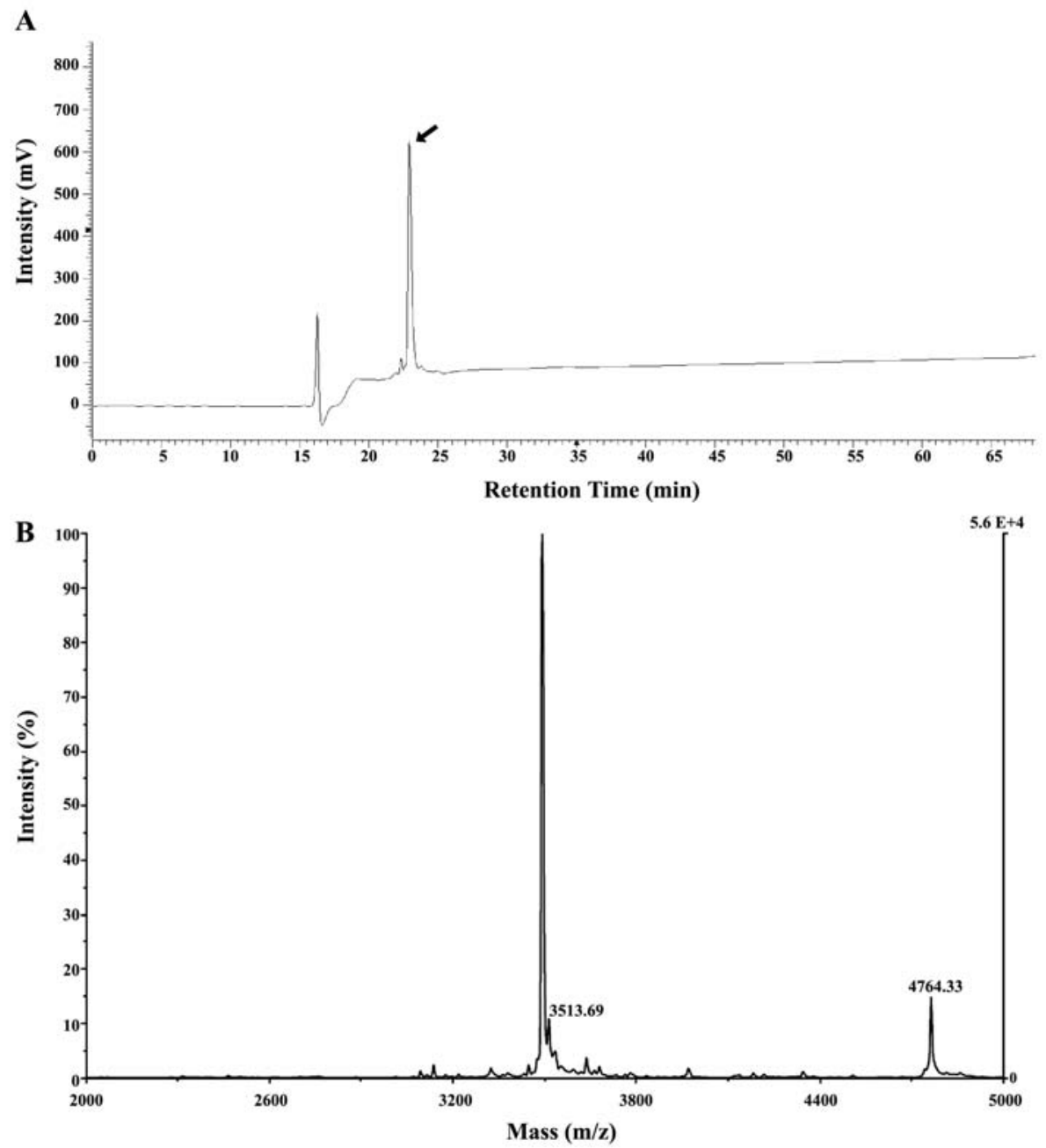

Figure 1. A: $\mathrm{C}_{18}$ reverse-phase chromatography profile of the bacteriocin from $W$. cibaria 860106 . The peak of bacteriocin activity, observed at $23 \mathrm{~min}$, is indicated by a black arrow. B: MALDI-TOF mass spectrum analysis of the purified bacteriocin from $W$. cibaria 860106 shows the mass $(\mathrm{m} / \mathrm{z})$ of $3492.21 \mathrm{Da}$ 
A

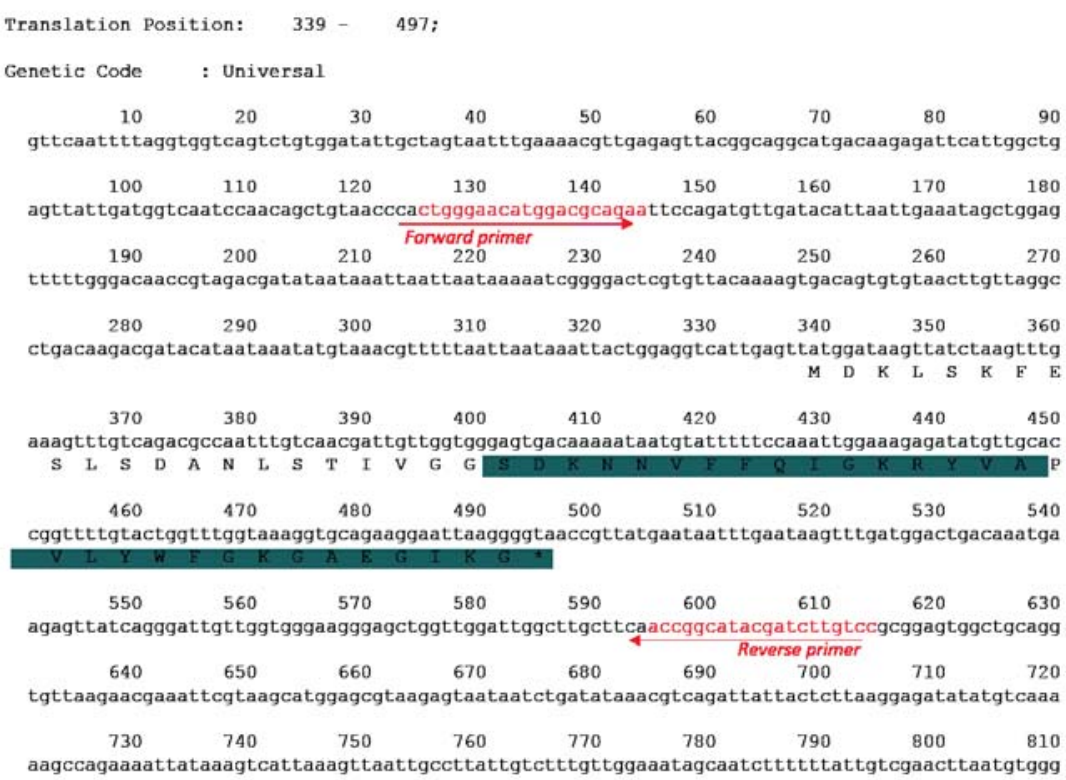

B

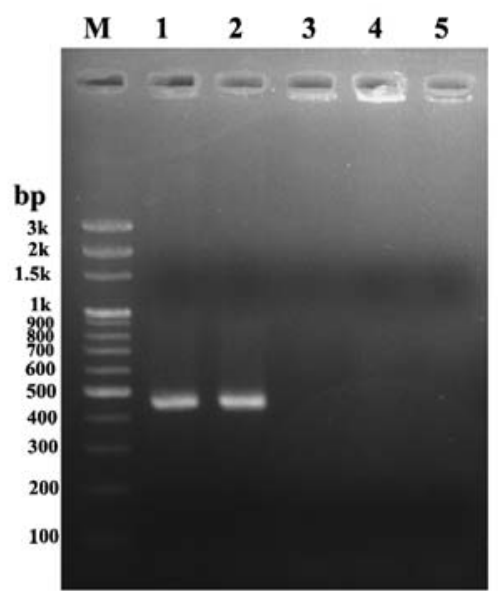

Figure 2. A: Nucleotide sequence of the weissellicin 110-encoding gene and the deduced amino acid sequence. An asterisk indicates the translation stop site. The mature weissellicin 110 peptide is highlighted in grey. B: Ethidium bromide-stained $2 \%$ agarose gel of PCR products using weissellicin 110-encoding gene-specific primers. Lane M, 100-bp DNA ladder; Lane 1, W. cibaria 860106; Lane 2, W. cibaria 110; Lane 3, W. hellenica 4-7; Lane 4, W. cibaria I042209; Lane 5, W. cibaria I042210

Primers specific for the weissellicin 110 encoding gene were designed and used for PCR amplification. Single fragments of the expected size (490 bp) were amplified from the genomic DNA of $W$. cibaria strains 860106 and 110. However, no PCR product was generated from $W$. hellenica 4-7 or from $W$. cibaria strain I042209 or I0422010 (Figure 2B). Sequencing of the PCR product derived from $W$. cibaria 860106 yielded nucleotide sequences identical to those obtained from the genomic sequence. Additionally, the same single $490 \mathrm{bp}$ fragment was obtained from each of the other $27 \mathrm{~W}$. cibaria strains tested by the PCR assay.

\section{Discussion}

Weissellicin 110 is the only bacteriocin reported in Weissella cibaria. We successfully determined the nucleotide sequence of weissellicin 110 and the information was used to detect the distribution of the bacteriocin.

Primers specific for the $W$. cibaria weissellicin 110 encoding gene were generated to perform PCR. As shown in (Figure 2B), a single amplified PCR productwas obtained from each of the W. cibaria 860106 and 110 strains. To verify the specificity of the PCR for the weissellicin 110 encoding gene, different Weissella species (with and without the demonstrated ability to produce bacteriocins) were used as negative controls. Notably, PCR of genomic DNA from strains $W$. hellenica 4-7 (a weissellicin L producer) and $W$. cibaria I042209 and I042210 (not weissellicin producers) did not yield amplicons. We propose that PCR for the presence of the weissellicin 110 encoding gene may be useful in screening of $W$. cibaria strains for their capability of producing the weissellicin.

Another $27 \mathrm{~W}$. cibaria strains with known bacteriocin producing ability were screened by PCR; all 27 yielded products of the expected size upon amplification with primers corresponding to sequences flanking the weissellicin 110 encoding gene. These strains had been isolated from any of four different Taiwanese fermented foods (or from the raw materials used in those fermentations). The results suggested that each of these strains encodes a bacteriocin identical to weissellicin 110. It is interesting that these independent strains isolated from different fermented foods and geographical locations $(14,19)$. However, the role of these weissellicin 110 producing $W$. cibaria strains in the fermented foods or the related vegetables remains elusive.

In conclusion, our study provided the full amino acid sequences of weissellicin 110 and the nucleotide sequences encoding the bacteriocin. These sequences 
permitted the design of primers for use in a PCR-based screen for the presence of the weissellicin 110 encoding gene. Our results showed that weissellicin 110 producing $W$. cibaria strains are widely distributed in Taiwanese fermented foods.

\section{Acknowledgments}

The authors would like to thank the Ministry of Science and Technology for financially supporting this study under MOST Grant No.103-2313-B-130-001 to Huichung Wu and No. 103-2313-B-130-002 to Yi-sheng Chen.

\section{References}

1. Chahad OB, El Bour M, Calo-Mata P, Boudabous A, BarrosVelàzquez J. Discovery of novel biopreservation agents with inhibitory effects on growth of food-borne pathogens and their application to seafood products. Res Microbiol. 2012;163:44-54. DOI:10.1016/j.resmic.2011.08.005

2. Chang $\mathrm{CH}$, Chen YS, Yanagida F. Isolation and characterisation of lactic acid bacteria from yan-jiang (fermented ginger), a traditional fermented food in Taiwan. J Sci Food Agric. 2011;91:1746-1750. DOI: 10.1002/jsfa.4364

3. Zendo T. Screening and characterization of novel bacteriocins from lactic acid bacteria. Biosci Biotechnol Biochem. 2013;77:893-899. DOI: 10.1016/0300-9084(88)90206-4

4. Dimov S, Ivanova P, Harizanova N. Genetics of bacteriocins biosynthesis by lactic acid bacteria. A review. Biotechnol Biotechnol Eq. 2005;19:4-10. DOI: 10.1080/13102818.2005. 10817270

5. Nishie M, Nagao J, Sonomoto K. Antibacterial peptides "bacteriocins": an overview of their diverse characteristics and application. .Biocontrol Sci. 2012;17:1-16. DOI:http://doi.org /10.4265/bio.17.1

6. Michiels J, Dirix G, Vanderleyden J, Xi C. Processing and export of peptide pheromones and bacteriocins in Gram-negative bacteria. Trends Microbiol. 2001;9:164-168. DOI: http://dx.doi.org/10.1016/S0966-842X (01)01979-5

7. Gálvez A, Abriouel H, López RL, Ben Omar N. Bacteriocinbased strategies for food biopreservation. Int J Food Microbiol. 2007;120:51-70. DOI:10.1016/j.ijfoodmicro. 2007.06.001

8. Srionnual S, Yanagida F, Lin LH, Hsiao KN, Chen YS. Weissellicin 110, a newly discovered bacteriocin from Weissella cibaria 110, isolated from plaa-som, a fermented fish product from Thailand. Appl Environ Microbiol. 2007;73:2247-2250. DOI: 10.1128/AEM.02484-06

9. Papagianni M; Papamichael EM. Purification, amino acid sequence and characterization of the class IIa bacteriocin weissellin A, produced by Weissella paramesenteroides DX. Bioresour Technol. 2011;102:6730-6734. DOI: 10.1016/j.bio rtech.2011.03.106

10. Masuda Y, Zendo T, Sawa N, Perez RH, Nakayama J, Sonomoto K. Characterization and identification of weissellicin $\mathrm{Y}$ and weissellicin $\mathrm{M}$, novel bacteriocins produced by Weissella hellenica QU 13. J Appl Microbiol. 2012;112:99108. DOI: 10.1111/j.1365-2672.2011.05180.x

11. Leong KH, Chen YS, Lin YH, Pan SF, Yu B, Wu HC, Yanagida
F. Weissellicin L, a novel bacteriocin from sian-sianzih-isolated Weissella hellenica 4-7. J Appl Microbiol. 2013;115:70-76. DOI: 10.1111/jam.12218

12. Chen YS, Lee YS, Wu HC, Chiang CM, Pan SF, Leong KH. Identification of nucleotide sequence involved in Weissellicin L production. Springer Plus. 2014;3:617. DOI: 10.1186/21931801-3-617

13. Lan WT, Chen YS, Yanagida F. Isolation and characterization of lactic acid bacteria from Yan-dong-gua (fermented wax gourd), a traditional fermented food in Taiwan. J Biosci Bioeng. 2009;108:484-487. DOI: 10.1016/j.jbiosc.2009.06. 009

14. Chen YS, Wu, HC, Wang CM, Lin CC, Chen YT, Jhong YJ, Yanagida F. Isolation and characterization of lactic acid bacteria from pobuzihi (fermented cummingcordia), a traditional fermented food in Taiwan. Folia Microbiol.2013;58:103-109. DOI: 10.1007/s12223-012-0188-4

15. Chen YS, Wu HC, Pan SF, Lin BG, Lin YH, Tung WC, Li YL, Chiang CM, Yanagida F. Isolation and characterization of lactic acid bacteria from yan-taozih (pickled peaches) in Taiwan. Ann Microbiol. 2013;63:607-614. DOI: 10.1007/s13213-0120510-Z

16. Yang R, Johnson MC, Ray B. Novel method to extract large amounts of bacteriocins from lactic acid bacteria. Appl Environ Microbiol. 1992;58:3355-3359.

17. Chen YS, Wang YC, Chow YS, Yanagida F, Liao CC, Chiu CM.Purification and characterization of plantaricin Y, a novel bacteriocin produced by Lactobacillus plantarum 510. Arch Microbiol. 2014;196:193-199. DOI: 10.1007/s00203-0140958-2

18. Chen YS, Yu CR, Ji SH, Liou MS, Leong KH, Pan SF, Wu HC, Lin YH, Yu B, Yanagida F. Enterocin T, a novel class IIa bacteriocin produced by Enterococcus sp. 812. Arch Microbiol. 2013;195:655-660. DOI: 10.1007/s00203-0130917-3

19. Chen YS, Wu HC, Yanagida F. Isolation and characteristics of lactic acid bacteria isolated from ripe mulberries in Taiwan. Braz J Microbiol. 2010;41:916-921. DOI: http://dx.doi.org/ $10.1590 / \mathrm{S} 1517-83822010000400010$ 